\title{
SUBSTITUIÇÃO DO NITROBENZENO PELO ÓLEO DE SOJA COMO UMA PROPOSTA PARA O ENSINO DO MÉTODO DE VOLHARD EM ANÁLISE QUANTITATIVA
}

\author{
Bárbara Helena S. Vieira ${ }^{a}$, Roberta Barra P. Lã ${ }^{b}$, José G. Rocha Jra, Otavio R. Lãa e Cristina M. Barra ${ }^{a}$ ** \\ aDepartamento de Química, Instituto de Ciências Exatas, Universidade Federal Rural do Rio de Janeiro, 23.897-000 Seropédica \\ - RJ, Brasil

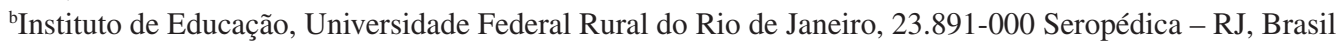

Recebido em 03/04/2017; aceito em 04/05/2017; publicado na web em 11/07/217

\begin{abstract}
REPLACEMENT OF NITROBENZENE BY SOYBEAN OIL AS A PROPOSAL FOR THE TEACHING OF THE VOLHARD METHOD IN QUANTITATIVE ANALYSIS. Volhard method is extensively employed in the teaching laboratories of classical quantitative analysis to chloride determination and is based on the reaction of $\mathrm{Ag}^{+}$with $\mathrm{Cl}^{-}$and $\mathrm{Ag}^{+}$residual titration with $\mathrm{SCN}^{-}$. Its drawback is that $\mathrm{SCN}^{-}$reacts with the $\mathrm{AgCl}_{(\mathrm{s})}$ initially formed. Nitrobenzene is used to avoid this stripping, but it is toxic to the environment and causes problems in final disposal. This work proposed the use of soybean oil as an alternative to nitrobenzene. The traditional and modified methods were applied in the standardization of $0.05 \mathrm{~mol} \mathrm{~L}^{-1} \mathrm{KSCN}$ solution employing $0.05000 \mathrm{~mol} \mathrm{~L}^{-1}$ $\mathrm{NaCl}$ standard and the proposed modification did not affect the results obtained $(\alpha=0.05)$. No significant differences were observed in the variances and $\mathrm{NaCl}$ recovery percentage in the titration of aliquots of 5.00 to $15.00 \mathrm{~mL}$ of solution of this salt. The recovery percentages obtained with soybean oil on the titration of synthetic $0.07000 \mathrm{~mol} \mathrm{~L}^{-1} \mathrm{NaCl}$ samples diluted 1:1 to 1:100 were equivalent and even better than with nitrobenzene. The chloride determination in samples of saline, whey cheese, sea water and olive brine obtained by the modified method was satisfactory when compared to the official method with relative errors below $3 \%$.
\end{abstract}

Keywords: back titration; argentometry; waste treatment; green chemistry.

\section{INTRODUÇÃO}

A titrimetria (ou volumetria) de precipitação é um método quantitativo clássico baseado em reações que produzem compostos de baixa solubilidade, bastante útil na determinação de vários íons que ocorrem em solução aquosa quando existe uma técnica adequada para a estimativa do ponto final, como as potenciométricas ou pelo uso de indicadores químicos. Diversas universidades brasileiras incorporaram em suas aulas práticas de Química Analítica métodos precipitimétricos, dentre os quais têm se destacado os métodos de Mohr, Fajans e Volhard.

Apesar de estes três métodos empregarem o nitrato de prata, que é um reagente de alto custo e elevada toxicidade, eles têm sido abordados pelas seguintes razões: a alta velocidade de formação de precipitados com $\mathrm{O} \mathrm{Ag}^{+}$; a grande variedade de analitos que podem ser determinados, como halogenetos, $\mathrm{SCN}^{-}, \mathrm{CN}^{-}, \mathrm{CNO}^{-}$, mercaptanas, ácidos graxos e vários ânions inorgânicos bivalentes e trivalentes; utilização como métodos de referência no controle de qualidade de diferentes tipos de amostras. ${ }^{1,2}$ Além disso, é comum comparar e contrastar estes métodos nas disciplinas de análise química quantitativa clássica, pois isto permite introduzir questões relevantes que devem ser consideradas no desenvolvimento dos métodos titrimétricos de uma forma simples e direta, corroborando, ao mesmo tempo, os conceitos abordados na teoria de equilíbrio químico.

Para ilustrar este último ponto, os métodos de Mohr e Fajans, por exemplo, realizam a titulação direta do analito e diferem apenas no mecanismo de atuação dos indicadores (Tabela 1).

$\mathrm{O}$ primeiro método emprega íons $\mathrm{CrO}_{4}{ }^{2-}$ como indicador, que precipita com os íons $\mathrm{Ag}^{+}$nas imediações do ponto de equivalência, enquanto que o de Fajans utiliza um indicador de adsorção (fluoresceína, diclorofluoresceína, rodamina $6 \mathrm{G}$, eosina, etc) cuja forma desprotonada compete com o analito pelo sólido formado, adsorvendo-se ao mesmo somente quando a concentração de analito for suficientemente baixa. ${ }^{3,4}$ Ambos os métodos devem ser empregados apenas na titulação de soluções com pH na faixa de 6,5 - 10,5 (ou mais estreita, dependendo da amostra) para evitar a hidrólise básica do indicador $(\mathrm{pH}<6,5)$ ou a precipitação do titulante como $\mathrm{Ag}(\mathrm{OH})$ $(\mathrm{pH}>10,5),{ }^{2}$ conforme as reações representadas na Figura 1.

Contudo, a faixa de $\mathrm{pH}$ utilizada nos métodos de Mohr e Fajans tem a desvantagem de precipitar diversos sais de prata pouco solúveis a partir de vários ânions, como $\mathrm{PO}_{4}^{3-}, \mathrm{AsO}_{4}^{3-}, \mathrm{S}^{2-}, \mathrm{SO}_{3}{ }^{2-}, \mathrm{CO}_{3}{ }^{2-}$, $\mathrm{C}_{2} \mathrm{O}_{4}{ }^{2-}$, etc, ${ }^{5}$ o que constituiria uma fonte de erro caso estes ânions estejam presentes na solução que se pretende determinar apenas um halogeneto, por exemplo. Outros ânions, tais como $\mathrm{CN}^{-}$e $\mathrm{SO}_{4}{ }^{2-}$,

Tabela 1. Principais diferenças que ocorrem entre os métodos de Mohr, Fajans e Volhard

\begin{tabular}{lcccc}
\hline Método & Titulação & Titulante & Mecanismo de indicação & pH de trabalho \\
\hline Mohr & Direta & $\mathrm{AgNO}_{3}$ & precipitação & $6,5-10,5^{\mathrm{a}}$ \\
Fajans & Direta & $\mathrm{AgNO}_{3}$ & adsorção & $6,5-10,5^{\mathrm{a}}$ \\
Volhard & Retorno & $\mathrm{KSCN}$ & complexação & $<0$ \\
\hline
\end{tabular}

${ }^{a}$ Esta faixa pode ser mais estreita, dependendo da concentração dos reagentes ou da presença de outras substâncias. Titulações de amostras contendo amônia, por exemplo, devem ser realizadas em pHs abaixo de 7,2 para que não ocorra a formação de complexos amin-prata. 
Hidrólise do cromato:

$$
2 \mathrm{CrO}_{4}^{2-}+\mathrm{H}_{2} \mathrm{O}=\mathrm{Cr}_{2} \mathrm{O}_{7}^{2-}+2 \mathrm{OH}^{-}
$$

(indicador de Mohr)

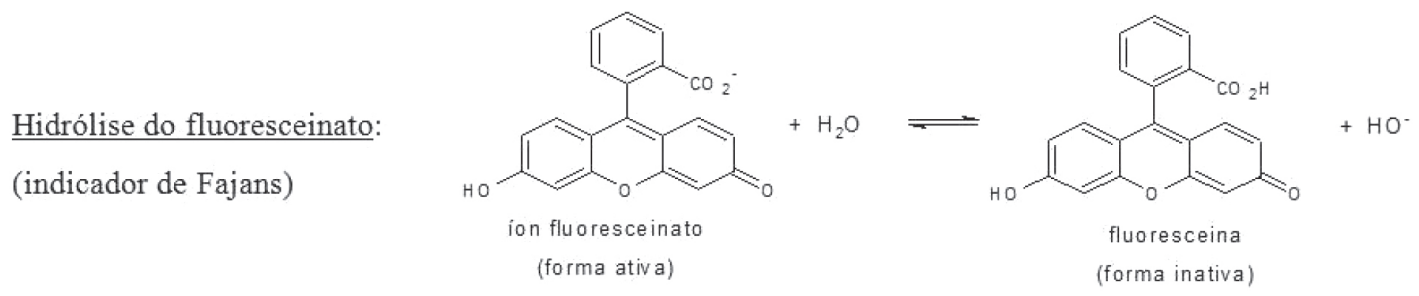

Precipitação do titulante: $\quad \mathrm{Ag}^{+}+\mathrm{HO}^{-} \rightleftharpoons \mathrm{Ag}(\mathrm{OH})_{(\mathrm{s})}$

Figura 1. Reações de hidrólise que ocorrem com o indicador de Mohr e Fajans (exemplificado pelo fluoresceinato) em pH menor do que 6,5 e reação de precipitação da prata como hidróxido de prata em pH acima de 10,5

também são interferentes, pois formam complexos metálicos com o íon $\mathrm{Ag}^{+}$. Cátions polivalentes, como $\mathrm{Al}^{3+}, \mathrm{Fe}^{3+}, \mathrm{Zn}^{2+}, \mathrm{Sn}^{2+}$, etc, devem estar ausentes porque precipitam como sais básicos contendo cloreto. Além disso, os cátions $\mathrm{Pb}^{2+}, \mathrm{Ba}^{2+} \mathrm{e} \mathrm{Sr}^{2+}$ também interferem, pois formam cromatos pouco solúveis com o indicador provocando erro na titulação. ${ }^{6}$

Tais interferências não ocorrem no método de Volhard, em que o meio reacional fortemente ácido impede a precipitação e a complexação dos ânions de caráter básico com a prata, tais como os citados acima. ${ }^{5}$ Igualmente, não interferem os cátions $\mathrm{Cu}^{2+}, \mathrm{Cd}^{2+}, \mathrm{Fe}^{3+}, \mathrm{Zn}^{2+}$, $\mathrm{Mn}^{2+}, \mathrm{Co}^{2+}, \mathrm{Ni}^{2+}$, etc, a menos que produzam soluções coloridas em concentração capaz de dificultar a visualização do ponto final.

O método de Volhard exemplifica uma titulação de retorno, em que o analito $\left(\mathrm{X}^{-}\right)$dissolvido é precipitado após a adição de uma quantidade conhecida de solução padrão de $\mathrm{AgNO}_{3}$ em meio fortemente ácido, com posterior titulação da prata residual por uma solução de tiocianato, formando o AgSCN (Figura 2). O indicador utilizado é o $\mathrm{Fe}^{3+}$, que forma um complexo vermelho-sangue $\left(\mathrm{FeSCN}^{2+}\right)$ nas imediações do ponto de equivalência da titulação.

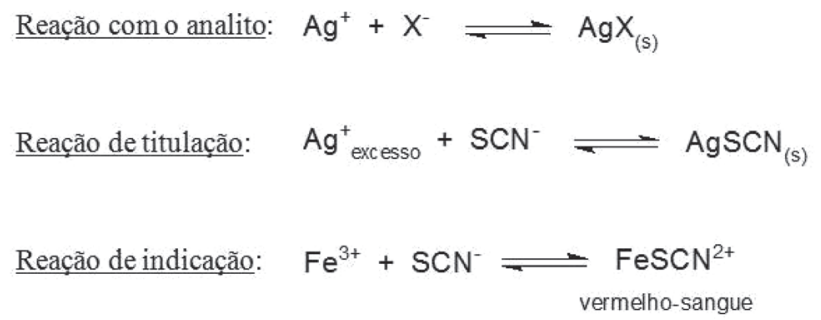

Figura 2. Reações do íon $\mathrm{Ag}^{+}$com o analito $\left(\mathrm{X}^{-}\right)$e com o titulante $\left(\mathrm{SCN}^{-}\right)$ e reação de indicação do ponto final da titulação que ocorrem no método de Volhard

Além de eliminar as interferências, o meio fortemente ácido tem a função de evitar a formação de hidróxi-complexos de $\mathrm{Fe}^{3+}$, além de prevenir a precipitação do indicador como hidróxido férrico, cuja coloração catanho-escura dificultaria a visualização do ponto final.

O método de Volhard tem sido empregado como método de referência na determinação de cloreto na análise de diversos tipos de amostras como carnes e derivados (AOAC O. M. 935.47), ${ }^{7}$ cerveja (AOAC O. M. 940.18), ${ }^{7}$ laticínios (AOAC O. M. 935.43), ${ }^{7}$ óleos lubrificantes e combustíveis (ASTM E 776), ${ }^{8}$ plantas (AOAC O. M. 915.01), ${ }^{7}$ peixes e produtos marinhos (AOAC O. M. 937.09), ${ }^{7}$ vinhos (AOAC O. M. 966.10), ${ }^{7}$ reagentes químicos (ASTM E 291), ${ }^{8}$

$$
\begin{array}{ll}
\mathrm{Fe}^{3+}+\mathrm{HO}^{-} \rightleftharpoons & \mathrm{FeOH}^{2+} \\
\mathrm{FeOH}^{2+}+\mathrm{HO}^{-} \rightleftharpoons & \mathrm{Fe}(\mathrm{OH})_{2}^{+} \\
\mathrm{Fe}(\mathrm{OH})_{2}^{+}+\mathrm{HO}^{-}= & \mathrm{Fe}(\mathrm{OH})_{3(\mathrm{~s})} \\
\text { castanho-escuro }
\end{array}
$$

Figura 3. Reações de formação dos hidróxi-complexos de $\mathrm{Fe}^{3+}$ e precipitação do $\mathrm{Fe}(\mathrm{OH})_{3}$ evitadas pela acidificação da solução titulada

etc. Contudo, para estas determinações, este método apresenta como um inconveniente o fato de a solubilidade do $\mathrm{AgCl}(1,34 x$ $10^{-5} \mathrm{~mol} \mathrm{~L}^{-1}, 25^{\circ} \mathrm{C}$ ) ser cerca de 13 vezes maior que a do $\mathrm{AgSCN}$ $\left(1,05 \times 10^{-6} \mathrm{~mol} \mathrm{~L}^{-1}, 25^{\circ} \mathrm{C}\right)$, permitindo a reação do tiocianato com uma fração do $\mathrm{AgCl}$ precipitado pelo $\mathrm{AgNO}_{3}$, segundo a reação

$$
\mathrm{AgCl}_{(\mathrm{s})}+\mathrm{SCN}^{-} \rightleftharpoons \mathrm{AgSCN}_{(\mathrm{s})}+\mathrm{Cl}^{-}
$$

Esta reação ocorre em extensão significativa perto do ponto de equivalência na titulação da prata residual, fazendo com que seja observado um ponto final evanescente, ocasionando uma adição excessiva de tiocianato e, consequentemente, um erro sistemático significativo que inviabiliza esta determinação. ${ }^{9}$

Para prevenir a reação do cloreto de prata com o tiocianato, foi proposto a remoção do $\mathrm{AgCl}$ por filtração. ${ }^{9-10}$ Contudo, apesar do excelente resultado proporcionado por este procedimento, o aumento do tempo requerido para a análise, aliado a mais uma fonte de erro inserida no método (a filtração) tem desestimulado o uso desta técnica. Para evitar a filtração, foi proposta a adição de compostos orgânicos de baixa solubilidade em água como éteres, ${ }^{11}$ benzeno e tolueno, ${ }^{12} \mathrm{e}$ o nitrobenzeno. ${ }^{13}$ Estes líquidos imiscíveis fazem com que o cloreto de prata seja extraído para a interface, coagulando com o mesmo, de modo a removê-lo da solução aquosa.

Dentre os compostos orgânicos recomendados, o nitrobenzeno tem sido extensivamente utilizado para este fim. Porém, o mesmo é muito tóxico tanto à saúde humana quanto ao meio ambiente. Ele pode ser absorvido pelas vias respiratória e dérmica, atuando como depressor do sistema nervoso central e apresenta ação meta-hemoglobinizante, provocando cianose. Em exposições crônicas pode produzir irritação ocular, lesões de baço e fígado e quadro de anemia. ${ }^{14}$ Portanto, o rejeito laboratorial tem que ser acondicionado e encaminhado para o descarte adequado.

Este trabalho tem como proposta a modificação do método de Volhard para a determinação de cloreto em amostras comercial e ambiental tanto para fins de ensino, bem como para aplicações 
diversas, fazendo a substituição do nitrobenzeno pelo óleo de soja. A potencialidade do óleo de soja como um agente aglutinador do $\mathrm{AgCl}$ está em sua baixa miscibilidade com a água, semelhantemente ao nitrobenzeno. Porém, o uso do óleo de soja seria bastante vantajoso pelo fato de apresentar baixa toxicidade, ser biodegradável e por ser saponificável, permitindo tanto o seu descarte como o tratamento da solução titulada para reaproveitamento de prata. Adicionalmente, o custo de 1,0 L de nitrobenzeno, após levantamento de preço realizado com alguns fornecedores, pode variar de $\mathrm{R} \$ 90,00$ a $\mathrm{R} \$ 160,00$, o que torna o óleo de soja uma alternativa bem mais econômica, pois o custo de 1,0 L no mercado varia de $\mathrm{R} \$ 3,50$ a $\mathrm{R} \$ 5,00$.

\section{PARTE EXPERIMENTAL}

\section{Materiais}

Os reagentes comerciais utilizados foram: $\mathrm{NaCl}$ (Vetec, 99\%); $\mathrm{AgNO}_{3}$ P.A. (Plat-lab); KSCN (Cinética, 98,5\%) e $\mathrm{K}_{2} \mathrm{CrO}_{4}$ (Vetec, $99,5 \%$ ). A amostra de água do mar foi coletada na Barra da Tijuca - Rio de Janeiro. O soro de queijo Minas foi coletado em amostra de queijo de produzido na UFRRJ, campus Seropédica-RJ. O soro fisiológico e a salmoura de azeitona foram obtidos no comércio local de Seropédica-RJ. Todas as amostras foram acondicionadas em frascos de polietileno e guardadas em geladeira até o momento das análises.

\section{Preparação dos padrões}

Inicialmente, foram preparadas duas soluções de $\mathrm{NaCl}$ de concentrações $0,05000 \mathrm{~mol} \mathrm{~L}^{-1} \mathrm{e} 0,07000 \mathrm{~mol} \mathrm{~L}^{-1}$ para serem empregadas como padrões primários, mediante a dissolução de massa suficiente de $\mathrm{NaCl}$ em água destilada e posterior diluição a 2000,0 mL, em balão volumétrico. Posteriormente, preparou-se 1000,0 mL de uma solução de $\mathrm{AgNO}_{3}$ de concentração $0,05 \mathrm{~mol} \mathrm{~L}^{-1}$ pela dissolução de $8,49 \mathrm{~g}$ deste reagente em água destilada. Esta solução foi padronizada titulando $10,00 \mathrm{~mL}$ da solução de $\mathrm{NaCl} 0,05000 \mathrm{~mol} \mathrm{~L}^{-1}$, empregando $1,0 \mathrm{~mL}$ de $\mathrm{K}_{2} \mathrm{CrO}_{4} 5 \% \mathrm{~m} / \mathrm{v}$ como solução indicadora (método de Mohr). A concentração molar obtida para a esta solução de $\mathrm{AgNO}_{3}$ foi de $0,04669 \mathrm{~mol} \mathrm{~L}^{-1}$.

A seguir, preparou-se uma solução de $\mathrm{KSCN}$ de concentração 0,05 mol L-1 dissolvendo 9,72 g de KSCN em água destilada o suficiente para 2000,0 mL de solução. A determinação da concentração molar exata (padronização) do KSCN foi feita pelo método de Volhard com o nitrobenzeno (método oficial) e com o óleo de soja (método modificado), utilizando como padrão a solução de $\mathrm{NaCl}$ $0,05000 \mathrm{~mol} \mathrm{~L}^{-1}$.

\section{Padronização da solução de KSCN com os métodos de Volhard oficial e modificado}

Os métodos de Volhard oficial e o modificado foram comparados mediante as concentrações obtidas para a solução de KSCN $0,05 \mathrm{~mol} \mathrm{~L}^{-1}$ após proceder a sua padronização com estes métodos. Na padronização com o método oficial, uma alíquota de $10,00 \mathrm{~mL}$ da solução padrão de $\mathrm{NaCl} 0,05000 \mathrm{~mol} \mathrm{~L}^{-1}$ foi transferida para um frasco erlenmeyer de $250,0 \mathrm{~mL}$, ao qual foram adicionados $25,00 \mathrm{~mL}$ da solução de $\mathrm{AgNO}_{3}$ 0,04669 mol L-1, 5,0 mL de ácido nítrico $6 \mathrm{~mol} \mathrm{~L}^{-1}, 1,0 \mathrm{~mL}$ de solução saturada de alumém férrico e 2,0 mL de nitrobenzeno. $\mathrm{O}$ erlenmeyer com esta mistura foi agitado para que o precipitado de $\mathrm{AgCl}$ ficasse impregnado com o nitrobenzeno. Posteriormente, esta solução foi titulada com a solução de KSCN $0,05 \mathrm{~mol} \mathrm{~L}^{-1}$, sob agitação, até o aparecimento de tom avermelhado, devido à formação de complexo entre o tiocianato $\mathrm{e} o \mathrm{Fe}^{3+}$.
O mesmo procedimento foi realizado para a padronização da solução de KSCN pelo método modificado, porém empregando 2,0 mL de óleo de soja como substituto para o nitrobenzeno. Vale ressaltar que, como o óleo de soja é menos denso que o nitrobenzeno, é conveniente agitar vigorosamente a solução contendo o $\mathrm{AgCl}$ neste método para que o precipitado fique completamente impregnado no óleo.

Foram realizadas 10 réplicas para cada método. O cálculo da concentração molar exata da solução de KSCN pelos métodos de Volhard oficial e modificado foi realizado a partir da equação 1

$$
\bar{M}=\frac{(M V)_{\mathrm{AgNO}_{3}}-(M V)_{\mathrm{NaCl}}}{\bar{V}}
$$

em que: $\bar{M}$ e $\bar{V}$ são a concentração, em mol L-1, e o volume, em mL, médios do titulante (KSCN), respectivamente; $M$ é a concentração, em mol L ${ }^{-1}$, e $V$ é o volume, em $\mathrm{mL}$, dos padrões destacados nesta equação, respectivamente.

Com o intuito de estimar as concentrações de cloreto nas soluções tituladas que podem ser quantificadas pelos métodos de Volhard oficial e modificado, foram realizados dois experimentos: a titulação de alíquotas de 5,00, 10,00 e 15,00 mL da solução padrão de $\mathrm{NaCl}$ $0,07000 \mathrm{~mol} \mathrm{~L}^{-1}$ e a titulação de $10,00 \mathrm{~mL}$ deste padrão de $\mathrm{NaCl}$ diluído nas proporções padrão:solução: $1: 1 ; 1: 2 ; 1: 4 ; 1: 5 ; 1: 7 ; 1: 10$; $1: 20 ; 1: 33 ; 1: 50 ; 1: 67 ;$ e $1: 100$. Estes experimentos foram realizados em triplicata.

\section{Determinação de cloreto em amostras reais}

Os métodos de Volhard oficial e modificado foram empregados para a determinação de cloreto em amostras de água do mar, soro fisiológico, soro de queijo Minas e de salmoura de azeitona. Para tal, foram analisadas alíquotas de $10,00 \mathrm{~mL}$ de solução-amostra diluídas na proporção 1:20 (amostra:solução). Foram realizadas 7 réplicas para cada análise.

\section{RESULTADOS E DISCUSSÃO}

A estimativa da concentração molar exata real $(\mu)$ da solução de KSCN obtida pelos métodos de Volhard oficial (A) e modificado (B), assim como de outros parâmetros populacionais foi realizada mediante o uso de técnicas de estatística inferencial. O teste $t$ de student foi utilizado para determinar $\mu_{A}$ e $\mu_{B}$ dentro do intervalo de confiança (IC), a um nível de significância $(\alpha)$ de 0,05 , aplicando a equação 2. Os resultados obtidos para os dois métodos estão apresentados na Tabela 2 .

$$
\text { IC para } \mu=\bar{M} \pm t_{\text {critico }} \frac{s}{\sqrt{N}}
$$

em que: $\mu$ é a concentração molar real $\left(\mu_{A}\right.$ ou $\left.\mu_{B}\right) ; \bar{M}$ é a concentração molar média das amostras $\left(\bar{M}_{A}\right.$ ou $\left.\bar{M}_{B}\right)$; $\mathrm{t}_{\text {crítico }}$ é o valor crítico de student; $s$ é o desvio-padrão da amostra $\left(s_{A}\right.$ ou $\left.s_{B}\right)$; e $N$ é o número de determinações.

A homogeneidade das variâncias $\left(\mathrm{s}^{2}\right)$ dos métodos de Volhard oficial e modificado foi avaliada empregando o teste estatístico F, em que o valor de $\mathrm{F}_{\text {calculado }}$ (equação 3 ) foi comparado com o valor de $\mathrm{F}_{\text {crítico }}$

$$
F_{\text {calculado }}=\frac{s_{A}^{2}}{s_{B}^{2}}
$$

em que $s_{A}^{2}$ e $s_{B}^{2}$ são as variâncias do método oficial e modificado, respectivamente. Como o valor de $\mathrm{F}_{\text {calculado }}$ foi menor que o $\mathrm{F}_{\text {crítico }}$ 
Tabela 2. Parâmetros estatísticos e intervalos de confiança (IC), em mol L-1, para a concentração molar real, $\mu$, da solução de KSCN após a sua padronização com os métodos de Volhard oficial e modificadoa

\begin{tabular}{lcc}
\hline Parâmetros estatísticos & $\begin{array}{c}\text { Volhard oficial } \\
\text { (nitrobenzeno) }\end{array}$ & $\begin{array}{c}\text { Volhard modificado } \\
\text { (óleo de soja) }\end{array}$ \\
\hline Molaridade média & 0,04991 & 0,04990 \\
Erro padrão(\%) & 0,0000377 & 0,0000268 \\
Desvio padrão & 0,00012 & 0,000085 \\
Variância $^{\mathrm{b}}$ & $1,42 \times 10^{-8}$ & $7,2 \times 10^{-9}$ \\
Mínimo & 0,04978 & 0,04978 \\
Máximo & 0,05009 & 0,05005 \\
IC para $\mu^{\mathrm{c}}$ & $0,04991 \pm 0,00009$ & $0,04990 \pm 0,00006$ \\
\hline
\end{tabular}

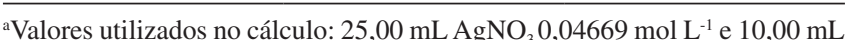
$\mathrm{NaCl} 0,05000 \mathrm{~mol} \mathrm{~L}^{-1}$; bem mol${ }^{2} \mathrm{~L}^{-2}$; 'obtido pela equação 2 , com $t_{c}=2,96$ (para $\alpha=0,05, \mathrm{gl}=9$ )

(Tabela 3), não houve diferença significativa na homogeneidade das variâncias entre os dois métodos ao nível de confiança de $95 \%$. Portanto, as precisões estimadas para os dois métodos não diferiram estatisticamente entre si.

O teste $\mathrm{t}$ student foi aplicado entre as concentrações médias da solução de KSCN obtidas pelos métodos oficial e modificado, para avaliar se a diferença entre elas seria decorrente de erros aleatórios. Neste caso, o teste estatístico empregado partiu da hipótese de que as médias das duas concentrações $\left(\mu_{\mathrm{A}} \mathrm{e} \mu_{\mathrm{B}}\right)$ são iguais (hipótese nula, $\left.\mathrm{H}_{\mathrm{o}}: \mu_{\mathrm{A}}=\mu_{\mathrm{B}}\right)$. Se esta suposição for verdadeira, a diferença entre $\mu_{\mathrm{A}} \mathrm{e}$ $\mu_{\mathrm{B}}$ é zero. Como as diferenças entre as médias oriundas de amostras retiradas de uma mesma população têm distribuição normal e as variâncias não diferiram significativamente, empregou-se a equação 4 para a determinação o parâmetro t.

$$
t_{\text {calculado }}=\frac{\bar{M}_{A}-\bar{M}_{B}}{s_{0} \sqrt{\frac{N_{A}+N_{B}}{N_{A} \times N_{B}}}}
$$

em que: $\bar{M}_{A}$ e $\bar{M}_{B}$ são as concentrações médias da solução de KSCN obtidas pelos métodos oficial e modificado, respectivamente; $N_{A}$ e $N_{B}$ são o número de réplicas executadas nos métodos oficial e modificado, respectivamente; $s_{0}$ é a estimativa de desvio padrão comum às duas molaridades $\left(\bar{M}_{A}\right.$ e $\left.\bar{M}_{B}\right)$, obtida pela equação 5 .
Tabela 3. Teste $\mathrm{F}$ entre as variâncias $\left(\mathrm{s}^{2}\right)$ e teste t entre as médias $(\bar{M})$ aplicados na comparação dos resultados referentes à concentração molar da solução de KSCN pelos métodos de Volhard oficial e modificado

\begin{tabular}{lcc}
\hline Variáveis estatísticas & $\begin{array}{c}\text { Volhard oficial } \\
\text { (nitrobenzeno) }\end{array}$ & $\begin{array}{c}\text { Volhard modificado } \\
\text { (óleo de soja) }\end{array}$ \\
\hline $\bar{M}$ & 0,04991 & 0,04990 \\
$\mathrm{~s}^{2}$ & $1,42 \times 10^{-8}$ & $7,2 \times 10^{-9}$ \\
$\mathrm{~F}_{\text {calculado }}<\mathrm{F}_{\text {crítico }}{ }^{a}$ & $1,98<3,18$ & \\
$\mathrm{t}_{\text {calculado }}<\mathrm{t}_{\text {crítico }}{ }^{b}$ & $-0,30<2,12$ & \\
\hline
\end{tabular}

${ }^{a}$ Foi utilizada a equação $3, \alpha=0,05$ e gl $=9$ (no numerador e denominador); ${ }^{\mathrm{b}}$ Foi utilizada a equação $4, \alpha=0,05 \mathrm{e} \mathrm{gl}=8$.

$$
s_{0}=\sqrt{\frac{\left(N_{A}-1\right) s_{A}^{2}-\left(N_{B}-1\right) s_{B}^{2}}{N_{A}+N_{B}-2}}
$$

Como o valor de $t_{\text {calculado }}$ foi menor que o $t_{\text {crítico }}$ (Tabela 3 ), as diferenças nas concentrações molares da solução de KSCN obtida na padronização pelos dois métodos são decorrentes de variações aleatórias (ao nível de confiança de 95\%) e, portanto, pode-se recomendar a utilização do óleo de soja como um substituto para o nitrobenzeno nesta padronização.

Nos experimentos realizados para avaliar se os métodos influenciariam no valor da concentração mínima de cloreto presente na solução titulada foi observado que não houve diferença na porcentagem de recuperação (\% Rec) de $\mathrm{NaCl}$ entre os métodos oficial e modificado quando se analisou as alíquotas de 5,00, 10,00 e 15,00 mL de solução padrão de $\mathrm{NaCl} 0,07000 \mathrm{~mol} \mathrm{~L}^{-1}$ (Tabela 4). Pela análise de variância dos resultados obtidos neste experimento (Tabela 5) nota-se que tanto a variação do volume pipetado como a variação do método empregado (i.e., a substituição do nitrobenzeno pelo óleo de soja) não provocaram alterações significativas nas porcentagens de recuperação de $\mathrm{NaCl}\left(\mathrm{F}_{\text {calculado }}<\mathrm{F}_{\text {crítico }}\right.$ e $\left.\mathrm{P}>0,05\right)$, mesmo após variar o volume e o método simultaneamente.

Nos experimentos realizados para avaliar a influência da concentração de cloreto nas percentagens de recuperação (\%Rec) do $\mathrm{NaCl}$ obtidas pelos métodos oficial e modificado (quando se analisou alíquotas de $10,00 \mathrm{~mL}$ do padrão de $\mathrm{NaCl}$ 0,07000 $\mathrm{mol} \mathrm{L}^{-1}$ após diluições nas proporções padrão:solução de 1:1, 1:2, 1:4, 1:5, 1:7, 1:10, 1:20, 1:33, 1:50, 1:67 e 1:100) foi observado que estas porcentagens foram próximas a 100\% nas diluições 1:1 e 1:2 para os métodos oficial e

Tabela 4. Porcentagens de recuperação (\%Rec) e porcentagens de recuperação média \pm desvio-padrão (\% $\overline{\operatorname{Re} c} \pm \mathrm{s})$ obtidas pelo método de Volhard oficial

\begin{tabular}{|c|c|c|c|c|}
\hline \multirow{2}{*}{ Volume (mL) de $\mathrm{NaCl}$} & \multicolumn{2}{|c|}{ Método oficial } & \multicolumn{2}{|c|}{ Método modificado } \\
\hline & $\% \operatorname{Rec}$ & $\% \overline{\operatorname{Re} c} \pm \mathrm{s}$ & $\% \operatorname{Rec}$ & $\% \overline{\operatorname{Re} c} \pm \mathrm{s}$ \\
\hline & 97,1 & & 98,3 & \\
\hline \multirow[t]{3}{*}{15,00} & 98,4 & $97,5 \pm 0,5$ & 97,0 & $97,5 \pm 0,7$ \\
\hline & 97,1 & & 97,0 & \\
\hline & 95,4 & & 97,0 & \\
\hline \multirow[t]{3}{*}{10,00} & 98,1 & $99,3 \pm 3,2$ & 97,5 & $97,2 \pm 0,2$ \\
\hline & 104 & & 97,2 & \\
\hline & 94,3 & & 95,0 & \\
\hline \multirow[t]{2}{*}{5,00} & 94,3 & $92,8 \pm 2,2$ & 100 & $95,5 \pm 3,0$ \\
\hline & 89,7 & & 91,6 & \\
\hline
\end{tabular}
(nitrobenzeno) e modificado (óleo de soja) após a titulação de diferentes alíquotas de solução de $\mathrm{NaCl}$ 0,07000 $\mathrm{mol} \mathrm{L}^{-1}$

Valores utilizados no cálculo: $25,00 \mathrm{~mL} \mathrm{AgNO}_{3} 0,04669 \mathrm{~mol} \mathrm{~L}^{-1}$, KSCN 0,05367 mol L-1 (método oficial) e KSCN 0,05327 mol L-1 (método modificado). 
Tabela 5. Tabela ANOVA de fator duplo para avaliação da influência do volume pipetado e do método empregado na \% Rec de $\mathrm{NaCl}$

\begin{tabular}{lcccccc}
\hline Fonte da variação & $\mathrm{SQ}$ & $\mathrm{gl}$ & $\mathrm{MQ}$ & $\mathrm{F}_{\text {calculado }}$ & valor-P & $\mathrm{F}_{\text {crítico }}$ \\
\hline Amostra & 3,404 & 1 & 3,404 & 0,630 & 0,450 & 5,318 \\
Colunas & 1,632 & 1 & 1,632 & 0,302 & 0,598 & 5,318 \\
Interações & 3,033 & 1 & 3,033 & 0,562 & 5,475 & \\
Dentro & 43,203 & 8 & 5,400 & & & \\
\hline Total & 51,272 & 11 & & & \\
\hline
\end{tabular}

$\alpha=0,05$.

modificado (Figura 1). Na proporção $1: 1$ a $\%$ Rec foi de $98,7 \pm 0,6 \mathrm{e}$ $97,5 \pm 0,9$ para o método oficial e modificado, respectivamente, enquanto que na proporção $1: 2$ a \% Rec foi de $102,9 \pm 1,9$ e 102,2 $\pm 1,2$, na mesma ordem apresentada.

Ao se empregar a equação 3 para a determinação de $\mathrm{F}_{\text {calculado, }}$, com exceção do caso no qual o padrão foi diluído na proporção 1:100, não foram observadas diferenças significativas entre as variâncias dos resultados obtidos pelos dois métodos nas diferentes diluições $\left(\mathrm{F}_{\text {calculado }}<\mathrm{F}_{\text {critico }}\right.$, que é igual a 19 para $\alpha=0,05$ e gl $=2$, no numerador e no denominador). Ao comparar os resultados entre estes grupos, utilizando o teste $\mathrm{t}$ (equação 4), foi possível notar que as recuperações obtidas pelo método oficial e modificado não diferiram estatisticamente somente nos casos em que o padrão foi diluído nas proporções 1:1 e 1:2 $\left(\mathrm{t}_{\text {calulado }}<\mathrm{t}_{\text {critico }}\right.$, que é igual a 2,78 para $\alpha=0,05$ e $\mathrm{gl}=4$ ).

Em ambos os métodos, as recuperações de $\mathrm{NaCl}$ nas diluições de 1:4 até 1:10 foram superestimadas, enquanto que nas diluições a partir de 1:20 foram abaixo de 100\%. Contudo, é possível notar que para todos estes casos a modificação do método de Volhard pelo uso do óleo de soja deu melhores resultados quando comparados ao nitrobenzeno (Figura 4), demonstrando que o metodo modificado apresenta aplicação para a titulação de amostras com uma faixa mais ampla de concentração em relação ao método oficial.

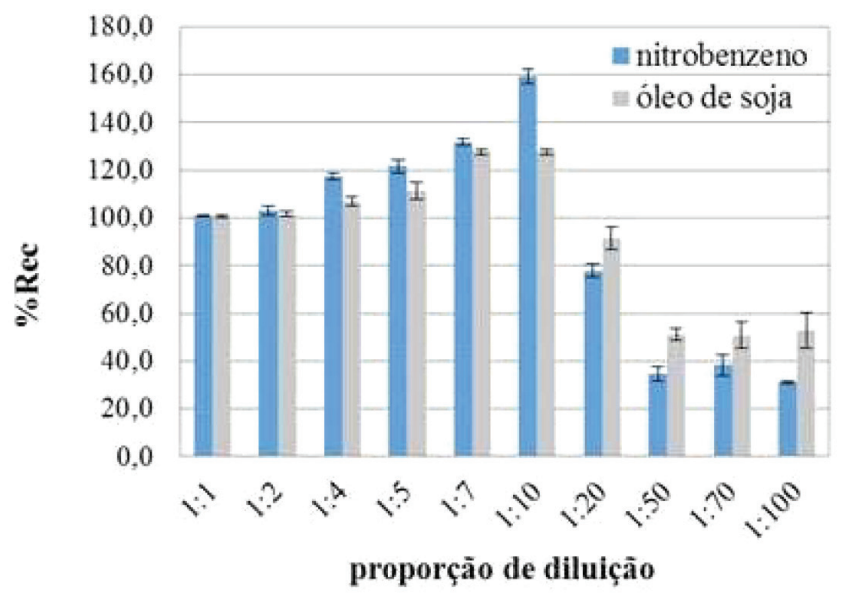

Figura 4. Porcentagens de recuperação \pm desvio-padrão $(\%$ Rec $\pm s)$ obtidas pelo método de Volhard oficial (nitrobenzeno) e modificado (óleo de soja) após a titulação de alíquotas de 10,00 $\mathrm{mL}$ de solução de $\mathrm{NaCl}$ 0,07000 $\mathrm{mol} \mathrm{L}^{-1}$ submetidas a diferentes diluições. Valores utilizados nos cálculos: $25,00 \mathrm{~mL}$ $\mathrm{AgNO}_{3}$ 0,04894 mol L $L^{-1}, \mathrm{KSCN}$ 0,04960 mol L-1 (método oficial) e KSCN $0,05008 \mathrm{~mol} \mathrm{~L}^{-1}$ (método modificado)

O aumento da \%Rec observado (Figura 4) com o aumento da diluição na faixa entre 1:4 e 1:10 é consequência do consumo de uma menor quantidade de titulante (solução de $\mathrm{KSCN}$ ), pois esta é uma titulação de retorno. Em outras palavras, entende-se que a coloração avermelhada que caracteriza o ponto final da titulação está ocorrendo mais precocemente à medida que diluição aumenta. Tal comportamento pode ser explicado se for considerada a formação de cloro-complexos de ferro $\left(\mathrm{FeCl}^{2+}\right.$ e $\left.\mathrm{FeCl}_{2}{ }^{+}\right)$na solução titulada, conforme esquematizado na Figura 5. Apesar de o cloreto ser precipitado como $\mathrm{AgCl}$, a concentração de cloreto livre será menor nos casos em que foram empregadas soluções mais diluídas deste íon devido ao maior excesso de prata na solução. Assim, pelo Princípio de Le Chatelier, a concentração do íon $\mathrm{Fe}^{3+}$ livre será maior para concentrações de cloreto mais baixas e, portanto, a coloração avermelhada ocasionada pela formação do complexo $\mathrm{FeSCN}^{2+}$ ocorrerá após a adição de menor quantidade de tiocianato.

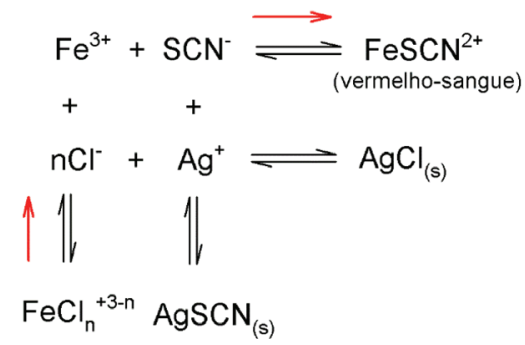

Figura 5. Esquema dos equilíbrios envolvidos na solução titulada pelos métodos de Volhard oficial e modificado. $n=1$ ou 2, para a formação dos cloro-complexos de ferro. Em vermelho está ilustrado o efeito da diminuição da concentração do $\mathrm{Cl}^{-}$nos equilíbrios de formação dos complexos $\mathrm{FeCl}_{n}^{+3-n}$ e FeSCN ${ }^{2+}$

Contudo, tal influência ocorre enquanto a diluição não for muito elevada, pois baixas recuperações foram observadas nos casos em que foram empregadas soluções de cloreto muito diluídas (faixa $1: 20-1: 100)$. Isto se justifica pelo fato de não haver precipitação quantitativa de $\mathrm{AgCl}$, uma vez que estará se trabalhando na faixa da solubilidade do $\mathrm{AgCl}$ em água, que é em torno de $1,0 \times 10^{-5} \mathrm{~mol} \mathrm{~L}^{-1}$. Consequentemente, haverá um aumento no consumo de KSCN e uma menor \%Rec.

A modificação do método de Volhard com o óleo de soja foi testada para a determinação do cloreto presente em amostras reais e os resultados obtidos foram comparados com os do método oficial mediante a aplicação dos testes $\mathrm{F}$ e t. $\mathrm{O}$ erro relativo percentual do método de Volhard modificado foi determinado supondo que os teores de cloreto obtidos pelo método oficial seriam os valores verdadeiros. Os teores de cloreto e os erros relativos percentuais obtidos estão listados na Tabela 6.

Como as variâncias dos resultados obtidos para as amostras de água do mar, soro fisiológico 1 e salmoura de azeitona diferiram significativamente entre os dois métodos ao nível de confiança de $95 \%$, seus resultados foram comparados empregando o teste $t$ representado na equação 6 , enquanto que para as amostras de soro fisiológico 2 e soro de queijo empregou-se o teste $t$ da equação 4. 
Tabela 6. Porcentagem média de cloreto \pm desvio-padrão, em g $100 \mathrm{~mL}^{-1}$ de $\mathrm{NaCl}$, nas amostras reais e o erro relativo percentual do método modificado

\begin{tabular}{lccc}
\hline Amostra & $\begin{array}{c}\text { Método oficial } \\
\text { (nitrobenzeno) }\end{array}$ & $\begin{array}{c}\text { Método modificado } \\
\text { (óleo de soja) }\end{array}$ & $\begin{array}{c}\text { Erro relativo } \\
\%\end{array}$ \\
\hline Água do mar & $3,528 \pm 0,006$ & $3,527 \pm 0,022$ & $-0,00860$ \\
Soro fisiológico 1 & $0,939 \pm 0,015$ & $0,940 \pm 0,006$ & 0,111 \\
Soro fisiológico 2 & $0,929 \pm 0,024$ & $0,919 \pm 0,018$ & $-1,009$ \\
Soro de queijo & $1,864 \pm 0,014$ & $1,810 \pm 0,014$ & $-2,912$ \\
Salmoura de azeitona & $3,997 \pm 0,005$ & $3,977 \pm 0,041$ & $-0,500$ \\
\hline
\end{tabular}

$\mathrm{N}=7$.

$$
t_{\text {calculado }}=\frac{\bar{M}_{A}-\bar{M}_{B}}{\sqrt{\frac{s_{A}^{2}}{N_{A}}+\frac{s_{B}^{2}}{N_{B}}}}
$$

em que: $\bar{M}_{A}$ e $\bar{M}_{B}$ são as concentrações médias da solução de KSCN obtidas pelos métodos oficial e modificado, respectivamente; $N_{A}$ e $N_{B}$ são o número de réplicas para os métodos oficial e modificado, respectivamente.

Pela aplicação do teste t, exceto a amostra de soro de queijo, a substituição do nitrobenzeno pelo óleo de soja não provocou variação significativa nos resultados ao nível de confiança de $95 \%$. Além disto, os erros relativos percentuais obtidos pelo método de Volhard modificado foram inferiores a 3\% para todas as amostras analisadas, podendo atingir valores muito baixos, demostrando que o óleo de soja fornece resultados comparáveis aos obtidos com o nitrobenzeno.

\section{CONCLUSÃO}

A modificação do método de Volhard pela utilização do óleo de soja como substituto do nitrobenzeno se apresenta como uma alternativa apropriada para a realização da padronização da solução de KSCN frente à solução padrão de $\mathrm{NaCl}$. Além disso, a determinação de cloreto em amostras sintéticas de $\mathrm{NaCl}$ fornece resultados semelhantes aos obtidos com o nitrobenzeno para diferentes concentrações deste analito e permite uma melhor estimativa do teor cloreto ao se empregar amostras mais diluídas. Na análise de amostras reais, os teores de $\mathrm{NaCl}$ obtidos diferiram estatisticamente somente na análise do soro de queijo. Porém, para todas as amostras analisadas o óleo de soja deu excelentes resultados quando comparados aos obtidos pelo nitrobenzeno, com erros relativos muito baixos.

Os resultados obtidos neste trabalho aliados à redução de riscos à saúde do manipulador e ao meio ambiente, à facilidade no tratamento dos rejeitos e ao seu baixo custo tornam o óleo de soja um substituto adequado para o nitrobenzeno na execução do método de Volhard.
Do ponto de vista didático, as vantagens da utilização do óleo de soja frente ao nitrobenzeno quando apresentadas em sala de aula, além de tornar evidente o compromisso do docente e da universidade com uma química mais verde, influencia na formação de profissionais mais comprometidos com esta ideia.

Pelas razões aqui apresentadas, o óleo de soja tem sido empregado na padronização do tiocianato e na determinação de cloreto em amostras sintéticas, pelo método de Volhard, nas aulas práticas de Química Analítica da UFRRJ.

\section{REFERÊNCIAS}

1. Andrade, J. C.; Alvim, T. R.; Chemkeys, 2009. http://www.chemkeys. com, acessada em Junho de 2017.

2. Kolthoff, I. M.; Stenger, V. A.; Volumetric analysis, vol. 2, $2^{\text {nd }}$ ed., Interscience Publ.: New York, 1964.

3. Baccan, N.; Andrade, J. C.; Godinho, O. E. S.; Barone, J. S.; Química Analítica Elementar, $3^{\text {a }}$ ed.; Edgar Blucher: Campinas, 2003, cap. 4.

4. Kolthoff, I. M.; Stenger, V. A.; Volumetric analysis, vol. 1, $2^{\text {nd }}$ ed., Interscience Publ:: New York 1964.

5. Abreu, D. G.; Costa, C. R.; Assis, M. D.; Iamamoto, Y.; Quim. Nova 2006, 29, 1381.

6. Vogel, A. I. In Química Analítica Qualitativa; Gimeno, A., ed.; $58^{\text {a }}$ ed., Mestre Ju: São Paulo, 1981, cap. 3.

7. Association of Official Analytical Chemists International, Official Methods of Analysis, 1997.

8. American Society for Testing and Materials; ASTM E 291 - Standard Test Methods for Chemical Analysis of Caustic Soda and Caustic Potash, 2003.

9. Swift, E. H.; Arcand, G. M.; Lutwack, R.; Meier, D. J.; Anal. Chem. 1950, 22, 306 .

10. Schoorl, N.; Pharm. Weekbl., Sci. 1905, 42, 233.

11. Rothmund, V.; Burgstaller, A.; Z. Anorg. Chem. 1909, 63, 330.

12. Stschigol, M. B.; Z. Anal. Chem. 1932, 91, 182.

13. Caldwell, J. R.; Moyer, H. V.; Ind. Eng. Chem. Anal. Ed. 1935, 7, 38.

14. Holder, J. W.; Toxicol. Ind. Health 1999, 15, 458. 